\title{
AN EXPERIMENTAL COMPARISON OF RANDOM FOREST AND SUPPORT VECTOR MACHINE FOR BEARING FAULT DIAGNOSIS: A MICRO ELECTRO MECHANICAL SENSOR APPROACH
}

\author{
Dr. H. S. KUMAR \\ Department of Mechanical Engineering, NMAM Institute of Technology, Nitte, Visvesvaraya \\ Technological University, Belagavi, Karnataka, India
}

\begin{abstract}
A sudden bearing failure leads to fatal accidents and results in loss of human life and increases the down time of the machine. Neural Network and Support Vector Machines (SVM) are widely used in rotating machinery fault diagnosis, while Random Forest $(\mathrm{RF})$ based on the ensemble learning method, is relatively unknown in this field. Currently, use of Micro Electro Mechanical Sensors (MEMS) for machinery fault diagnosis is receiving more attention as it is low cost, compact and portable. In this paper, vibration signals are collected from the Rolling Element Bearing (REB) using the MEMS sensor. Statistical features have been extracted from the wavelet packet coefficients of the vibration signals and used as input feature for the classification purpose. A framework for the comparison of RF and SVM is presented to identify the best classifier for bearing fault diagnosis. RF emerged as the best classifier based on the classification accuracy especially with a small training set leading to a promising tool for bearing fault diagnosis.

KEYWORDS: Rolling Element Bearing, Wavelet Transform, Feature Extraction, SVM \& Random Forest
\end{abstract}

Received: Apr 21, 2019; Accepted: May 12, 2019; Published: Jun 11, 2019; Paper Id.: IJMPERDJUN2019158

\section{INTRODUCTION}

Bearings are vital elements of rotary machinery right across the industrial sectors. The bearing failure leads to major breakdown resulting in untold and expensive down time that should be avoided at all costs. Hence, intelligently-based bearing failure diagnosis and prognosis should be an integral part of the asset maintenance and management activity in any industry using rotary machines [1-2]. Vibration analysis is superior as it reveals essential information about the condition of the machine [3]. The use of conventional piezoelectric accelerometers in vibration signal acquisition is costly affair because of their size, cost and price associated with the signal conditioning circuitry. Currently Micro electro Mechanical sensor (MEMS) is gaining significance as it is not only cheaper in price, but also provides the same results as that of conventional accelerometer. Jyoti K. Sinha [5] tested, integrated circuit piezoelectric (ICP) type accelerometers for its reliability. ICP accelerometer from three well known manufacturers was tested in the laboratory and he found accuracy of sinusoidal response measurement was satisfying. Jardine et al [6] discussed the use of multiple sensor \& different techniques for data fusion. They showcased the next generation diagnostic and prognostic methods for condition monitoring (CM). Uhlmann et al [7] presented an advanced wireless CM system for industrial units. Data acquisition, signal processing and classification are done using MEMS sensor and Raspberry Pi 2. Feldman et al [8] showed the use of MEMS sensor for in situ CM work and their proposed method assisted in early detection of failure, fault diagnosis and integrated 
diagnosis system.

Time and spectrum analysis methods are used in signal analysis to detect fault conditions. Time domain analysis provides basic description of the bearing health. Statistical features such as mean, standard deviation and Root Mean Square are used. In spectrum analysis, vibration data is represented as a function of time and it is not suitable for analyzing trainsient vibration signal, as it fails to reveal transient information such as impacts contained in it. These transient components contain vital information about the machinery defects. Hence, Wavelet Transform (WT) is commonly used to analyze them as it provides a variable window technique which uses different time intervals to analyze high frequency and low frequency components. It divides the data in to approximation and detail coefficients in variable scale providing efficient tool for non-stationary signal analysis than FFT [9]. WT works well with weak signals, denoising process and singularity detection [10].

Shimada et al [11] applied SVM to find the fault location in fixed components. They firstly used variations in the natural frequency of the body to train the SVM model, and then to detect fault location. The prime intention was to minimize the usage of sensors to acquire fault signal from the system. They found that this method adequately reduced the possibility of improper fault detection. Rojas et al [12] used SVM for bearing fault diagnosis. They used four conditions of bearing vibration signals in their work and concluded that SVM accuracy reduces with the reduction in the data size. Yan et al [13] used SVM for rotor failure diagnosis. They observed that the SVM was effective in identifying machinery failures particularly in complex operating conditions as there is limit for input parameters and the computation time is also small. Zhong et al [14] utilized SVM for the fault identification in gearbox. They designed a test setup to identify the most usual failures in the gearbox, such as misalignment, wear, and imbalance. They concluded that the SVMs are capable of recognizing various failure types precisely. Yang et al [15] investigated the usage of the random forests (RF) algorithm in machinery fault diagnosis. They developed a hybrid model by combining genetic algorithm with RF to enhance the output of the RF. The performance of the method is carried using induction motor vibration data and experimental data is used to validate the same. Ferenc et al [16] acquired the vibration signals from bearings and performed signal processing techniques like signal decomposition and signal denoising. They obtained eighteen statistical features from the healthy and faulty vibration signals utilizing wavelets. These features are given as input to the RF. RF yielded a classification accuracy of $99.51 \%$ for one tree. The accuracy was increased up to $99.81 \%$ when they increased the number of trees to eight. Yao et al [17] studied four types of rotor failure due to unbalancing, misalignment, surge, and bearing failure. They compared performances of decision tree (DT) and RF and concluded that RF resulted in higher classification accuracy in comparison to DT. To validate this method, the authors verified this method by comparing the results with SVM classifier. They found that RF is a valid method for fault diagnosis of rotors. Patel et al [18] established a RF classifier for multiclass problem of mechanical faults in an induction motor bearing. They compared the results of the RF with ANN and they concluded that RF is superior when compared to ANN. Seera et al [19] presented an intelligent system using RF and Fuzzy min-max neural network and identified it as FMM-RF. They used the developed model to ball bearing condition classification.

Literature reveals a number of such classifier like Decision Tree (DT), NN, SVM and naive Bayes. The model parameter such as penalty factor and kernel functions has an effect on SVM performance. The genetic algorithm and particle swarm optimization can be used for selecting model parameters which results in more computational time. Also, a single classifier will often emerge as a problem of low classification accuracy or over fitting. To address this issue, Random Forest is used which consists of several decision trees. Also, RF provides an opportunity to do diagnosis without 
performing feature selection task. This is because feature selection may lead to over- compressing the original data and as stated by Breiman and RF performance is superior when the data size is large. RF uses repetitive partitioning, resulting in several trees and then aggregate the results. Also, RF is not influenced by the surrounding noise. [3, 15-17].

In this paper, vibration signals are obtained using ADXL335 sensor for three states of the bearing i.e. New or unused (N), defect on Inner Race (IR) and defect on Outer Race (OR) at $355 \& 622 \mathrm{rpm}$ shaft speed and $1.7 \mathrm{kN}$ constant load. The raw signals are denoised and eleven statistical features have been obtained from the wavelet packet coefficients and these features are fed to RF and SVM. The classifier result will aid in selecting the best classifier in this work. Figure 1 demonstrate the proposed methodology used in this paper.

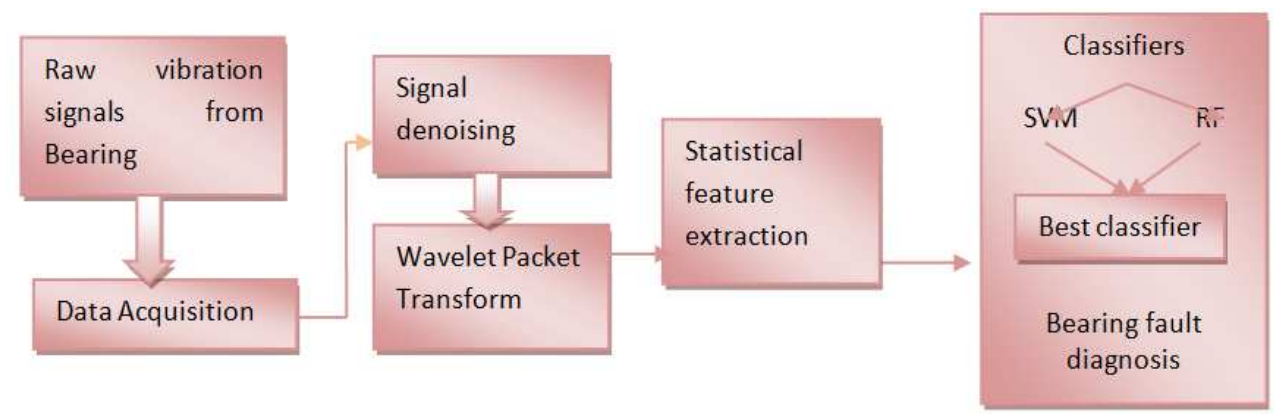

Figure 1: Proposed Methodology for Bearing Fault Diagnosis

\section{WAVELET TRANSFORM}

WT is categorized as Continuous Wavelet Transform (CWT), Discrete Wavelet Transform (DWT) and Wavelet Packet Transform (WPT). The CWT of a signal $x(t)$ can be obtained using a convolution operation between the signal $x(t)$ and the complex conjugate of the wavelet families, which is written as [9]

$$
\operatorname{cwt}(s, \tau)=\frac{1}{\sqrt{s}} \int_{-\infty}^{\infty} x(t) \psi^{*}\left(\frac{t-\tau}{s}\right) d t
$$

where $\psi^{*}(\bullet)$ is the complex conjugate of the scaled and shifted wavelet function $\psi(\bullet)$. It not only reduces redundancy of the data, but also provides quality information contained in the original signal. Dyadic scales ( $\left.\mathrm{s}=2, \tau=k 2^{\mathrm{j}}\right)$ is used to achieve this [9-10].

DWT is derived from the discritization of CWT and is expressed as

$$
D W T(j, k)=\left\langle x(t), \psi_{j, k}(t)\right\rangle=\frac{1}{\sqrt{2^{j}}} \int_{-\infty}^{\infty} x(t) \psi^{*}\left(\frac{t-k 2^{j}}{2^{j}}\right) d t
$$

where the symbol $\langle\bullet\rangle$ denotes inner product operation. Figure 2 shows the basic steps involved in the decomposition. 


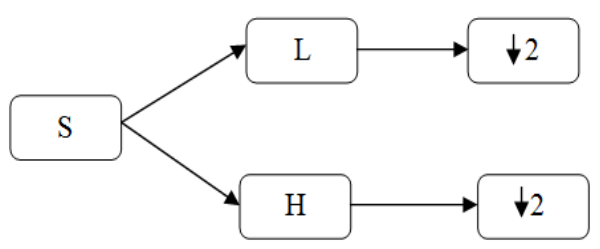

Figure 2: Basic Decomposition Step

In WPT, $\mathrm{W}$ is a function of three integers of $(i, j, k)$ defined as

$$
W_{j, k}^{n}(t)=2^{j / 2} W^{n}\left(2^{j} t-k\right)
$$

where integers $j$ and $k$ represent the index of scale and translation respectively, and the index $n$ is called the modulation parameter [9]. The first two - wavelet packet functions are scaling and mother wavelet functions represented as $\phi(t)$ and $\psi(t)$ respectively, given by

$$
\begin{aligned}
& W_{0,0}^{0}(t)=\phi(t) \\
& W_{0,0}^{1}(t)=\psi(t)
\end{aligned}
$$

where $n=2,3 \ldots$, the function can be defined by the following recursive relationships

$$
\begin{aligned}
& W_{0,0}^{2 n}(t)=\sqrt{2} \sum_{k} h(k) W_{j, k}^{n}(2 t-k) \\
& W_{0,0}^{2 n+1}(t)=\sqrt{2} \sum_{k} g(k) W_{j, k}^{n}(2 t-k)
\end{aligned}
$$

where ' $h(k)$ and $g(k)$ ' are the quadrature mirror filter associated with predefined scaling and mother wavelet functions [10]. The wavelet packet coefficient $W_{j, n, k}$ of the signal $f(t)$ can be computed using the following equation

$$
W_{j, n, k}=\left\langle f, W_{j, k}{ }^{n}\right\rangle=\int f(t) W_{j, k}^{n}(t) d t
$$

\section{EXPERIMENTAL SETUP AND MEMS SENSOR}

The raw signal is obtained from customized bearing test set up and is shown in figure 3 . The test setup has a shaft of $32 \mathrm{~mm}$ diameter and is supported between test and fixed bearing. The shaft rotor assembly is driven by a 3-phase induction AC motor. This unit has speed ratio of 2.25 which provides a variable speed i.e. 0 to1400 rpm. A hydraulic loading arrangement is made to apply vertical load on the bearing test. The ADXL335 is mounted on the test bearing unit and it is coupled to NI PCI 6221 Data Acquisition system (DAQ) hardware which in turn coupled to a computer through cables. The test rig is allowed to run for some time and the raw signals are obtained with the help of a DAQ board. The raw signals are sampled at 10,000 samples/sec using the customized LABVIEW (VI) programme and the signals are stored in the computer as.xls file [4].

The defects on bearing components are seeded through water jet machining, drilling, and indentation through hammering methods. In this work, defects were simulated on the IR and OR of 6206 bearing using indentation through 
hammer method. The parameters of SKF6206 bearing is shown in table 1. The ADXL 335 sensor provides analogue voltage as output which is proportional to acceleration by sensing changes. The sensor converts the capacitance changes due to acceleration into a voltage [4]. ADXL 335 sensor senses triaxial (X,Y and Z) direction accelerations. It is having an inbuilt temperature compensation circuitry, hence additional circuitry to reduce temperature effects is not required. ADXL 335 accelerometer has the following specifications: measurement range $\pm 3 \mathrm{~g}$, sensitivity: $300 \mathrm{mV} / \mathrm{g}$, frequency band width for $\mathrm{X}$ and $\mathrm{Y}$ terminal is $60 \mathrm{~Hz}$, and for $\mathrm{Y}$ terminal is $50 \mathrm{~Hz}$, power supply $-3 \mathrm{~V}$, Noise performance- $150 \frac{\mu \mathrm{g}}{\sqrt{\mathrm{Hz}}} \mathrm{rms}$

Figure 4 shows the photograph of the ADXL 335 MEMS accelerometer.

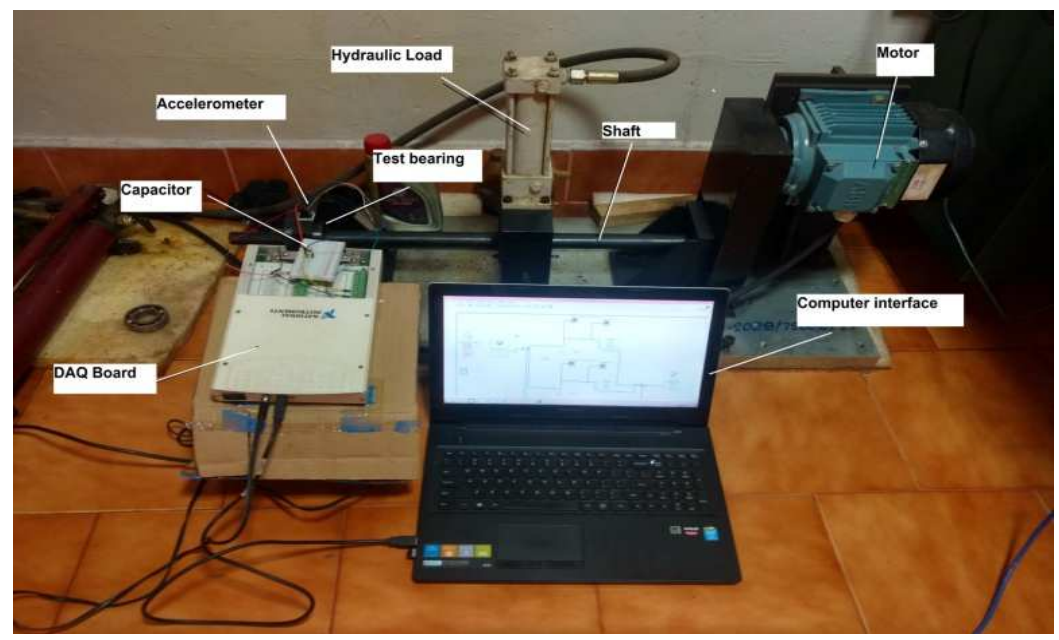

Figure 3: Photograph of Experimental Set Up
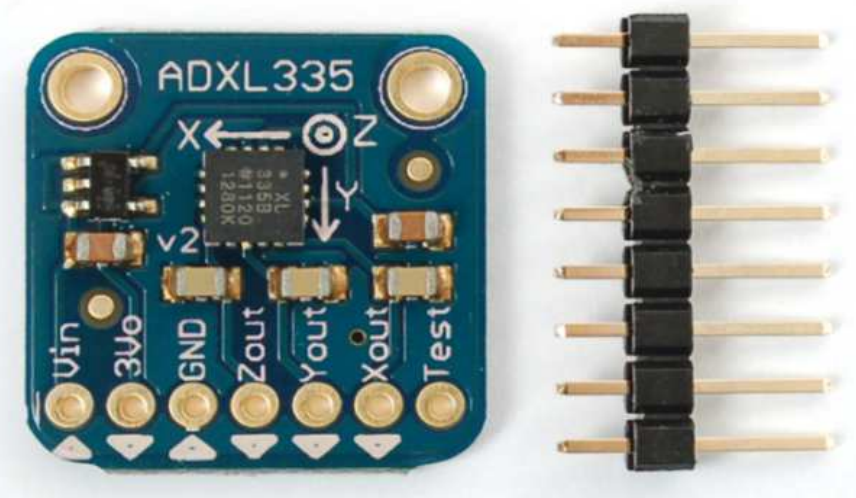

Figure 4: Photograph of ADXL335 MEMS Accelerometer

The 6206 SKF bearing specifications and its characteristic frequencies are indicated in table $1 \& 2$.

Table 1: SKF 6206 Bearing Parameter

\begin{tabular}{|c|c|c|c|c|c|}
\hline $\begin{array}{c}\text { Inner } \\
\text { Diameter }\end{array}$ & $\begin{array}{c}\text { Outer } \\
\text { Diameter }\end{array}$ & $\begin{array}{c}\text { Roller } \\
\text { Number }\end{array}$ & $\begin{array}{c}\text { Diameter } \\
\text { of Ball }\end{array}$ & $\begin{array}{c}\text { Angle of } \\
\text { Contact }\end{array}$ & $\begin{array}{c}\text { Pitch } \\
\text { Diameter }\end{array}$ \\
\hline 25 & 52 & 9 & 7.93 & 0 & 39 \\
\hline
\end{tabular}

Table 2: Characteristic Frequencies

\begin{tabular}{|c|c|c|}
\hline $\begin{array}{c}\text { Rotating Frequency } \\
(\mathbf{H z})\end{array}$ & $\begin{array}{c}\text { Ball Pass Frequency Inner Race } \\
(\mathbf{H z})\end{array}$ & $\begin{array}{c}\text { Ball Pass Frequency Outer Race } \\
(\mathbf{H z})\end{array}$ \\
\hline 23.33 & 128.41 & 87.65 \\
\hline
\end{tabular}




\subsection{Modification of ADXL335 Accelerometer for band Width Selection}

As per the specification of ADXL 335 accelerometer, the maximum band width available is $60 \mathrm{~Hz}$. In this work, the required maximum frequency band width is $128 \mathrm{~Hz}$ (as shown in table 2). This led to the modification of the existing commercial ADXL 335 accelerometer. The required band width is obtained by removing existing capacitor and replacing it with a new capacitor of value $0.03 \mu \mathrm{F}$ in series [20]. The vibration signals were acquired at $10 \mathrm{k}$ samples/sec for ten seconds. The 'N, IR and OR ' were collected at the operating load $1.7 \mathrm{kN}$ and varying shaft speeds.

\subsection{Wavelet Denoising}

It is hard to remove the noise effectively just by utilizing traditional filtration method. Also, these traditional denoising methods require the knowledge of the some technical parameters $[4,21]$. To overcome all these barriers, the wavelet based denoising is used. The customised MATLAB codes are used to denoise the raw signal acquired from the test rig $[4,22]$. Figure 5 and 6 shows the plot of raw and denoised vibration signals for 3 conditions of bearing and the energy values of the odd wavelet packet coefficients at level three considered in this work.

The energy and kurtosis values are computed using MATLAB codes. The kurtosis and Energy raw and denosed signals at $622 \mathrm{rpm}$ is shown in Table 3. Denosing is superior when energy values of the denoised signal are lower and kurtosis values are higher when compared to the raw vibration signal [9-10]. This trend is clearly observed in the table 3. The OR has highest kurtosis value and follows a descending sequence for IR and N. This is due to the fact that outer race is closer to ADXL 335 sensor and hence captures defect characteristic information effectively when compared to IR and N [24].

Table 3: Raw and Denoised Signal Energy and Kurtosis

\begin{tabular}{|c|c|c|c|c|c|}
\hline Sl. No. & Condition & $\boldsymbol{E}_{\text {raw }}$ & $\boldsymbol{E}_{\text {den }}$ & $\boldsymbol{K}_{\text {raw }}$ & $\boldsymbol{K}_{\text {den }}$ \\
\hline 1. & $\mathrm{~N}$ & 0.02 & 0.018 & 3.17 & 3.31 \\
\hline 2. & $\mathrm{IR}$ & 0.0608 & 0.055 & 5.15 & 7.80 \\
\hline 3. & $\mathrm{OR}$ & 0.08 & 0.064 & 4.37 & 46.73 \\
\hline
\end{tabular}

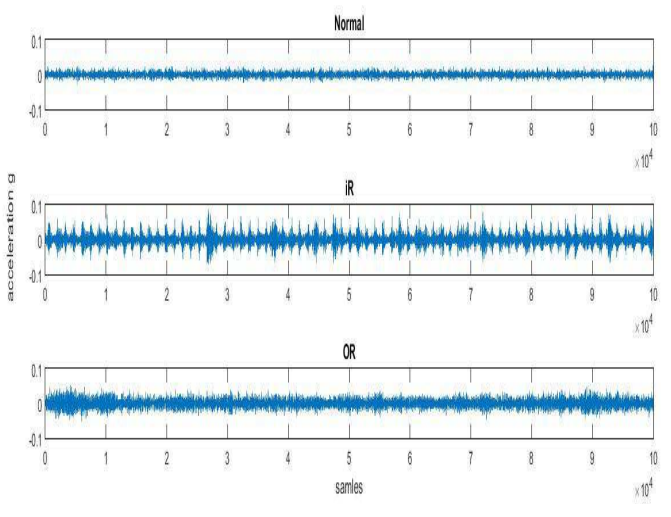

(a)Raw

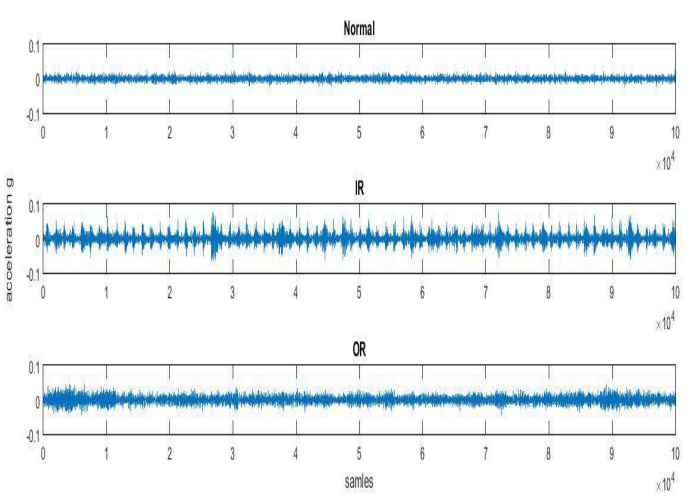

(b) Denoised

Figure 5: Plots of (a) Raw Signals and (b) Wavelet Denoised Vibration Signals for the Three Bearing Conditions: N, IR, and OR, at a Speed of 622 rpm and Load of $1.7 \mathrm{kN} \mathrm{[4]}$

\section{WAVELET TRANSFORM AND FEATURE EXTRACTION}

WT can be represented in CWT, DWT, and WPT [4]. Among these WPT is superior as it decomposes detailed coefficients of the signal, thus providing a solution to the limitation of DWT [9-10]. WPT is superior when compared to 
other WT methods; Hence, WPT is used in this work. The noise free signal is subjected to WPT and analysed up to 3 level using ' $\mathrm{db} 1$ ' wavelet using customized codes in MATLAB [22]. Figure 6 shows the energy values of the odd wavelet packet coefficients computed using MATLAB codes [22]. An ascending trend is observed for wavelet packet node $(3,7)$ which is not found in the reaming packet nodes. Hence, in this work statistical features are extracted from this wavelet packet node. The denoised vibration signal is split into 10 segments with each segment comprising of 10000 samples. Eleven statistical features have been extracted from each segment of the signal. Table 4 shows the list of statistical features used in this work [4].

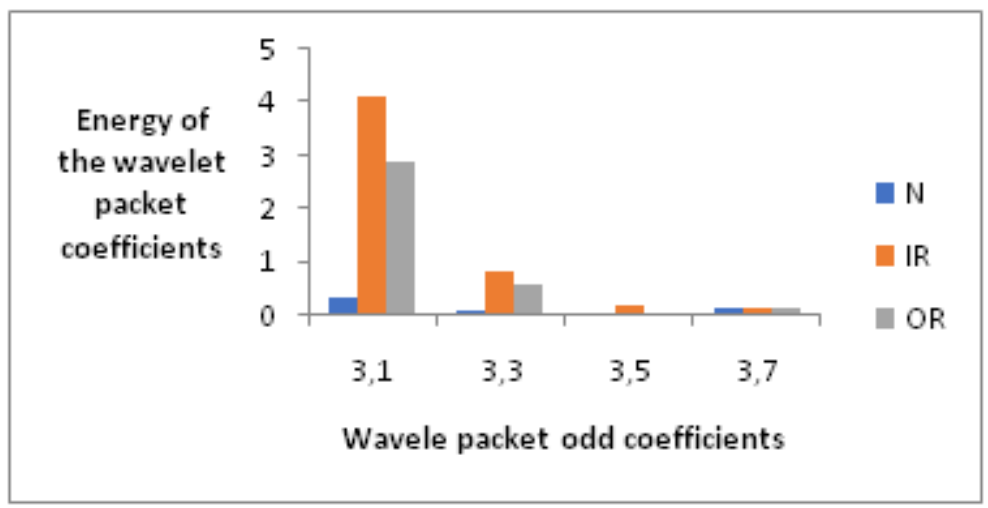

Figure 6: Energy Values of the Odd Wavelet Packet Coefficients

Table 4: List of Statistical Features

\begin{tabular}{|c|c|c|c|c|c|}
\hline $\begin{array}{l}\text { S1. } \\
\text { No. }\end{array}$ & Features & Formulae & $\begin{array}{l}\text { S1. } \\
\text { No. }\end{array}$ & Features & Formulae \\
\hline T1 & Mean & $\frac{\sum_{i=1}^{n} x_{i}}{n}$ & T7 & Variance & $\left(\frac{1}{N}\right) \sum_{n=1}^{N}(x(n)-\bar{x})^{2}$ \\
\hline $\mathrm{T} 2$ & RMS & $\sqrt{\left(\frac{1}{N}\right) \sum_{n=1}^{N} x(n)^{2}}$ & T8 & $\begin{array}{l}\text { Crest } \\
\text { Factor }\end{array}$ & $\mathrm{T} 4 / \mathrm{T} 2$ \\
\hline T3 & $\begin{array}{l}\text { Standard } \\
\text { deviation }\end{array}$ & $\sqrt{\left(\frac{1}{N}\right) \sum_{n=1}^{N}(x(n)-\bar{x})^{2}}$ & T9 & $\begin{array}{l}\text { Latitude } \\
\text { Factor }\end{array}$ & $\mathrm{T} 4 / \sqrt{T 1^{2}}$ \\
\hline $\mathrm{T} 4$ & $\begin{array}{l}\text { Peak- } \\
\text { Peak } \\
\text { value }\end{array}$ & $\max x(n)-\min x(n)$ & T10 & $\begin{array}{l}\text { Impulse } \\
\text { Factor }\end{array}$ & $\mathrm{T} 4 / \mathrm{T} 1$ \\
\hline T5 & Skewness & $\frac{\left(\left(\frac{1}{N}\right) \sum_{n=1}^{N}(x(n)-\bar{x})^{2}\right)^{3}}{\left(\sqrt{\left(\frac{1}{N}\right) \sum_{n=1}^{N}(x(n)-\bar{x})^{2}}\right)^{3}}$ & T11 & $\begin{array}{l}\text { Log-Log } \\
\text { Ratio }\end{array}$ & $\begin{array}{r}\left(\frac{1}{\log T 3}\right) \sum_{i=1}^{n}\left(\log \left|x_{i}\right|\right. \\
+1)\end{array}$ \\
\hline T6 & Kurtosis & $\frac{\left(\frac{1}{N}\right) \sum_{n=1}^{N}(x(n)-\bar{x})^{4}}{\left(\left(\frac{1}{N}\right) \sum_{n=1}^{N}(x(n)-\bar{x})^{2}\right)^{2}}$ & & & \\
\hline
\end{tabular}

\section{CLASSIFIERS}

Two machine learning techniques, namely, RF and SVM are used to check the diagnostic capability of acquired vibration signals.

\subsection{Random Forest}

$\mathrm{RF}$ is introduced by Breiman [24], uses decision tree (DT) type classification. It divides the original training set into a large number of subsets and from these subsets it forms DT by using bagging technique [24]. Bagging helps in minimizing the variance and over-fitting and also it improves the classification and regression models corresponding to classification accuracy and stability [24]. The bagging process randomly selects feature samples from the training set and 
decision trees have been constructed with the help of bootstrapping technique. All the tree classifier is termed a class predictor. The RF forms decisions by counting the votes of class predictors on each class and after that choosing the best suited class as far as the quantity of votes to it. Figure 7 shows the methodology adopted in RF.

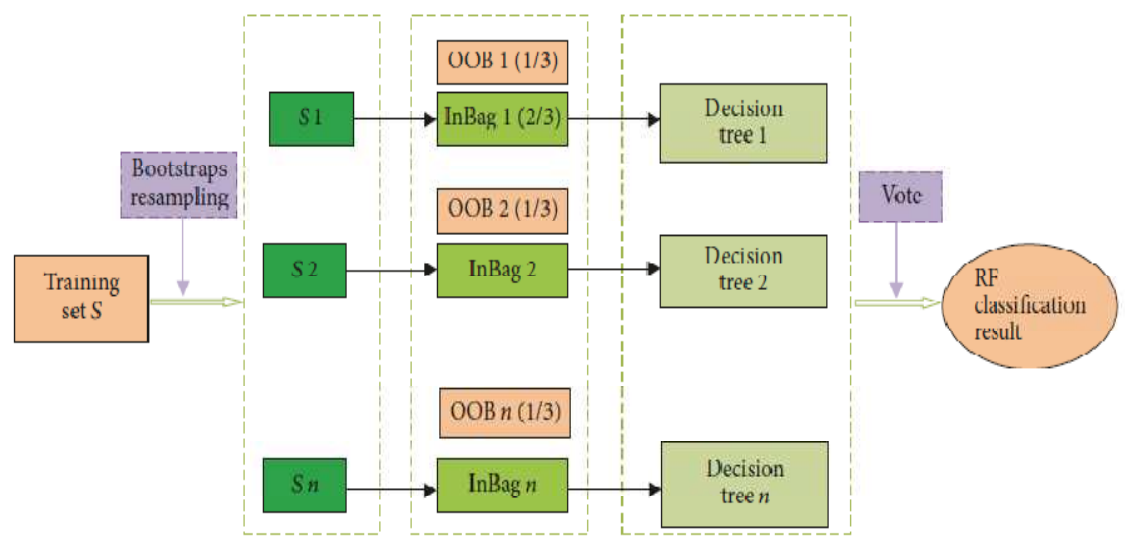

Figure 7: Work Flow of RF Algorithm [26]

The steps involved in the working of RF are explained as follows.

Table 5

\begin{tabular}{|c|l|}
\hline S1. No & \multicolumn{1}{|c|}{ Discription } \\
\hline 1 & $\begin{array}{l}\text { Consider a random sample of n observations from the data set with the replacement of m observations } \\
\text { using boot strap resampling method. In this, }(2 / 3)^{\text {rd }} \text { of samples will be selected which contain repetitive } \\
\text { samples and the remaining }(1 / 3)^{\text {rd }} \text { are termed as "out of bagging' (OOB). New random selection of cases } \\
\text { is performed for each constructed trees". [25-26]. }\end{array}$ \\
\hline 2 & $\begin{array}{l}\text { Step 2: Construct DT (maximum size without pruning) using the cases considered in the previous step. } \\
\text { In this, consider a subset of the total set of predictor variables every time that it is needed to split the } \\
\text { node. The predicted set variable is selected as random subset of the total available predictor variable. In } \\
\text { each split, some prediction results cannot be considered, but prediction results omitted in one split can be } \\
\text { used by other split in the same tree. }\end{array}$ \\
\hline 3 & $\begin{array}{l}\text { Step 3: Repeat step 1 and step 2 to generate forest i.e. large number of trees. } \\
\text { Step 4: Run the example through each tree in the forest and note the predicted value to score a case. Use } \\
\text { the predicted categories for each tree as "vote" for the best class, and decide the output based on the } \\
\text { majority votes gathered by the class [26]. }\end{array}$ \\
\hline
\end{tabular}

\subsection{Support Vector Machine}

SVM has been demonstrated successful in abnormality detection. SVM was presented by Vapnik. It streamlines a limiting curve by augmenting the separation of the nearest point to the limiting curve [12]. It is an effective technique for classification and prediction with a small number of observations. It is utilized as a part of machine fault diagnosis, bearing defect detections and diagnosis, and machine learning, since it indicates remarkable execution in modelling and generalizing when contrasted with other methods for example, neural network The ground characteristic of the SVM model is to outline the original nonlinear data into a high-dimensional feature space and a hyper plane is built to divide two classes' data and augment the margin of separation amongst itself and those points lying closest to the support vectors [ $23,26]$.

Let input data, $\mathrm{xi}(\mathrm{i}=1,2, \ldots, \mathrm{n}), \mathrm{n}$ is the total sample number. The samples are considered as positive and negative class designated as $\mathrm{yj}=1$ for positive class and $\mathrm{yi}=-1$ for negative class, respectively. In the case of linearly 
separating data, the hyper plane $\mathrm{f}(\mathrm{x})=0$ which divides the data sets is obtained by:

$$
f(x)=w^{T} x+b=\sum_{j=1}^{n} w_{j} x_{j}+b=0
$$

where, $\mathrm{w}$ is vector with dimension $\mathrm{n}$ and $\mathrm{b}$ is a scalar. The position of separating hyper plane is interpreted by scalar $b$ and vector $w$. The separating hyper plane is created by decision function made utilizing sign $f(x)$ to classify the input data into either positive class or negative class [27]. The separating hyper plane should hold the rule,

$$
y_{i} f\left(x_{i}\right)=y_{i}\left(w^{T} x_{i}+b\right) \geq 1 \text { for } i=1,2,3, \ldots . n
$$

From the geometry, the geometrical margin is observed to be $\left\|w^{2}\right\|$. Considering the noise with slack variable $\xi i$ and the error penalty $\mathrm{C}$, the ideal hyper plane separating the data can be attained as a solution to the below given optimization problem [27].

$$
\operatorname{Minimize}\left(\frac{1}{2}\right)\left\|w^{2}\right\|+C \sum_{i=1}^{n} \zeta_{i}
$$

Subjected to

$$
\begin{cases}y_{i}\left(w^{T} x_{i}+b \geq 1-\xi_{i}\right. & i=1 \ldots . . n \\ \xi_{i} \geq 0 & i=1 \ldots . . n\end{cases}
$$

where, $\xi_{i}$ is the separation between the boundary and the class $x_{i}$ that lying on the false side of the margin [27].

As shown in Figure 8, SVM works in a way that the dotted lines illustrate the margins whose separations is maximized, and in the middle of these margins, a boundary is placed between two points.

For the condition where the linear SVM does not produce adequate result, the non-linear SVM is used. Here the feature vector $x$ is produced by non-linear mapping $\emptyset(x)$, to a high dimension feature space, where the best hyper plane can be found. In non-linear SVM, the kernel functions are utilized to avoid over-fitting through which a complete non-linear mapping is performed. The equation for kernel function is given by,

$$
k\left(x_{i}, x_{j}\right)=\left\{\phi\left(x_{i}\right), \phi\left(x_{j}\right)\right\}
$$

Where, $\varnothing\left(x_{i}\right)$ and $\emptyset\left(x_{j}\right)$ are the inner products of the vector.

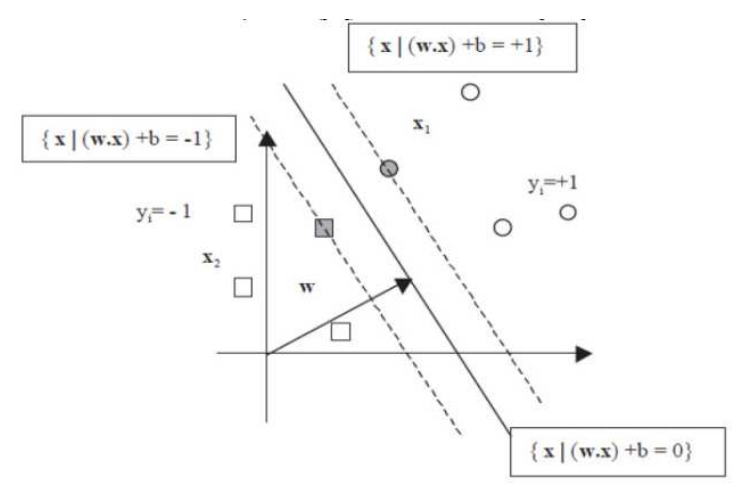

Figure 8: SVM Classification of Two Classes [26] 
The kernel functions used in SVM is represented in table 6.

Table 6: Formulation of Kernel Functions

\begin{tabular}{|l|c|}
\hline Kernel Function & Formula \\
\hline Linear & $x_{i}^{T} x_{j}$ \\
\hline Polynomial & $\left(\gamma x_{i}^{T} x_{j}+r\right)^{d}, \gamma>0$ \\
\hline RBF & $e^{\frac{\left\|x_{i}-x_{j}\right\|^{2}}{2 y^{2}}}$ \\
\hline
\end{tabular}

\section{RESULTS AND DISCUSSIONS}

This work estimates the diagnostic performance of vibration signal acquired using MEMS accelerometer using RF and SVM. The vibration signals were acquired at $1.7 \mathrm{kN}$ load, 355 and $622 \mathrm{rpm}$ shaft speed for three bearing conditions. Vibration signals were denoised and denoised signals were subjected to WPT. Eleven statistical features were extracted from the wavelet packet coefficients Therefore; the dimension of the feature vector is $60 \times 11$. These features were then divided into $42(70 \%)$ training data and 18 (30\%) testing data that were used in the construction of the RF and SVM models and testing scheme respectively.

To attain high classification results, some model parameters need to be set for optimal values for the classifier. In RF, there is only one parameter that affects the performance, i.e. number of trees. The SVM model to classify bearing fault diagnosis was constructed using the one-against-one method. The RBF kernel function was used in the SVM model. RF and SVM models were constructed and then the models were tested using the test data to predict the conditions of the bearings. All features (eleven in this work) were given as input to SVM model. The best classifier is identified based on the classifier results.

The classification accuracy on test data for RF and SVM is found to be $88.8 \%$ and $72.22 \%$ respectively as evident from the table 7. To clearly understand about the class wise classification accuracy on test data for RF and SVM, a confusion matrix is used. Table 7 gives insight about the prediction accuracy of fault cases considered such as N, IR and OR for both RF and SVM. The diagonal elements of the confusion matrix represent the correctly classified conditions. In this work, the first element (i.e. first row element) 6 samples are correctly classified as ' $N$ ', where as second element in the same row i.e. 1 is misclassified as 'IR'. Of the 18 samples, 16 samples are correctly classified by the RF with a classification accuracy of $88.8 \%$. Similar observations can be applied to the SVM confusion matrix. Thus 13 samples were correctly classified with a classification accuracy of $72.2 \%$.

Table 7: Classification Accuracies of RF and SVM

\begin{tabular}{|c|c|c|c|c|}
\hline Accelerometer & \multicolumn{2}{|c|}{ RF (\%) } & \multicolumn{2}{c|}{ SVM } \\
\hline & Training Accuracy & Test Accuracy & Training Accuracy & Test Accuracy \\
\hline MEMS & 90.4 & 88.8 & 73.8 & 72.22 \\
\hline
\end{tabular}

Table 8: Confusion Matrix of RF and SVM

\begin{tabular}{|c|c|c|c|c|c|c|}
\hline \multicolumn{3}{|c|}{ RF } & \multicolumn{3}{c|}{ SVM } & \\
\hline $\mathrm{N}$ & IR & OR & N & IR & OR & \\
\hline 6 & 1 & 0 & 5 & 1 & 0 & N \\
\hline 1 & 5 & 0 & 0 & 3 & 3 & IR \\
\hline 0 & 0 & 5 & 0 & 1 & 5 & OR \\
\hline
\end{tabular}


Performance of RF mainly depends on number of tress. RF provides better result with large number of trees; also it takes large computational time. Relation between the number of trees and test accuracy is presented in Table 9. When number of trees increased, until a high number, for example 200 or 300, no over-fitting was observed but a little rippling exists. Classification accuracy showcases an ascending trend up to 300 trees and then accuracy decreases even though the number of trees increases.

Table 9: Number of Trees and Corresponding Test Accuracy

\begin{tabular}{|c|c|}
\hline No. of Trees & Test Accuracy (\%) \\
\hline 100 & 77.8 \\
\hline 200 & 81.3 \\
\hline 300 & 88.8 \\
\hline 400 & 86.12 \\
\hline 500 & 67.8 \\
\hline 600 & 67.8 \\
\hline
\end{tabular}

\section{CONCLUSIONS}

Currently, SVM has gained progressively more importance while RF is relatively unfamiliar in bearing fault diagnosis area. In this work, REB fault diagnosis procedure was presented in which low cost ADXL335 MEMS accelerometer was used to acquire vibration signals. The performance of RF is compared with the existing SVM technique. The results demonstrated the superiority of the RF over SVM. RF is superior as it contains several decision trees which avoids the problems associated with single decision tree (DT) such as over fitting or low classification accuracy. Also, RF doesn't require feature selection task for fault diagnosis which reduces the computation time.

\section{ACKNOWLEDGMENT}

The author would like to thank the Condition Monitoring Research Lab for allowing me to use the Bearing test-rig to acquire the vibration signal for bearing fault diagnosis

\section{REFERENCES}

1. Mishra, R. C., and K. Pathak. Maintenance engineering and management. PHI Learning Pvt. Ltd., 2012.

2. Patil, M. S., Jose Mathew, and P. K. Rajendra Kumar. "Bearing signature analysis as a medium for fault detection: A review." Journal of Tribology 130.1 (2008), 014001-1 to 014001-7.

3. Han, Te, D. Jiang, D, Zhao, Q, Wang, L and Yin, K, "Comparison of random forest, artificial neural networks and support vector machine for intelligent diagnosis of rotating machinery." Transactions of the Institute of Measurement and Control 40.8 (2018): 2681-2693.

4. Kumar, H.S. Srinivasa Pai P., Sriram, N. S. Vijay G. S., “Rolling element bearing fault diagnostics: Development of health index", Proceedings of the Institution of Mechanical Engineers, Part C: Journal of Mechanical Engineering Science 2017, Vol. 231 issue. 21, pp. 3923-3939

5. Sinha, Jyoti K. "On standardisation of calibration procedure for accelerometer." Journal of Sound and Vibration 2 86.1-2 (2005): 417-427.

6. Jardine, Andrew KS, Daming Lin, and Dragan Banjevic. "A review on machinery diagnostics and prognostics implementing condition-based maintenance." Mechanical systems and signal processing 20.7 (2006): 1483-1510. 
7. Uhlmann, E., Laghmouchi, A., Hohwieler, E., \& Geisert, C. "Condition monitoring in the cloud." Procedia CIRP 38(2015): 53-57.

8. Feldman, J., Hanrahan, B. M., Misra, S., Fan, X. Z., Waits, C. M., Mitcheson, P. D., \& Ghodssi, R, "Vibration-based diagnostics for rotary MEMS." Journal of Microelectromechanical Systems 24.2 (2015): 289-299.

9. Dae-Sung, K., Ji-Hyeung, Y., \& Hong-Duk, M. (2016). Non-Destructive Stress Measurement of Civil Structural Steel Using Magnetic Anisotropy Sensor. International Journal of Civil, Structural, Environmental and Infrastructure Engineering Research and Development (IJCSEIERD) ISSN (P), 2249-6866.

10. Ahmed, A. S., \& Rani, P. R. (2017). A Study On Sensory Limitations Among Elderly, In The Selected Oldage Homes Of Hyderabad City. Available at SSRN 3094275.

11. Yan, Ruqiang, Robert X. Gao, and Xuefeng Chen. "Wavelets for fault diagnosis of rotary machines: A review with applications." Signal processing 96 (2014): 1-15.

12. Peng, Z. K., and F. L. Chu. "Application of the wavelet transform in machine condition monitoring and fault diagnostics: a review with bibliography." Mechanical systems and signal processing 18.2 (2004): 199-221.

13. Shimada, M., and A. Mita. "Damage assessment of bending structures using support vector machine." Smart Structures and Materials 2005: Sensors and Smart Structures Technologies for Civil, Mechanical, and Aerospace Systems. Vol. 5765, International Society for Optics and Photonics, 2005.

14. Rojas, Alfonso, and Asoke K. Nandi. "Practical scheme for fast detection and classification of rolling-element bearing faults using support vector machines." Mechanical Systems and Signal Processing 20.7 (2006): 1523-1536.

15. Yan, J., Ma, H., Li, W., \& Zhu, H. "Assessment of rotor degradation in steam turbine using support vector machine." Power and Energy Engineering Conference, 2009, APPEEC 2009. Asia-Pacific. IEEE, 2009.

16. Zhong, Jianhua, Zhixin Yang, and S. F. Wong. "Machine condition monitoring and fault diagnosis based on support vector machine." IEEE International Conference on, Industrial Engineering and Engineering Management (IEEM), 2010 IEEE, 2010.

17. Yang, Bo-Suk, Xiao Di, and Tian Han. "Random forests classifier for machine fault diagnosis." Journal of mechanical science and technology 22.9 (2008): 1716-1725.

18. Ferenc, G., Lutovac, M., Kvrgić, V., \& Stepanić, P, "A proposed approach to the classification of bearing condition using wavelets and random forests. "In 2nd Mediterranean Conference on Embedded Computing (MECO), IEEE, 2013.

19. Widodo, Achmad, and Bo-Suk Yang. "Support vector machine in machine condition monitoring and fault diagnosis." Mechanical systems and signal processing 21.6 (2007): 2560-2574.

20. Patel, Raj Kumar, and V. K. Giri. "Feature selection and classification of mechanical fault of an induction motor using random forest classifier." Perspectives in Science 8 (2016): 334-337.

21. Seera, Manjeevan, ML Dennis Wong, and Asoke K. Nandi. "Classification of ball bearing faults using a hybrid intelligent model." Applied Soft Computing 57 (2017): 427-435.

22. Chaudhury, Subimal Bikash, Mainak Sengupta, and Kaushik Mukherjee. "Vibration monitoring of rotating machines using MEMS accelerometer." International Journal of Scientific Engineering and Research 2.9 (2014): 1-1.

23. Хue, Y. J., Cao, J. X., Tian, R. F., \& Ge, $Q$ "Feature extraction of bearing vibration signals using autocorrelation denoising and improved Hilbert-Huang transformation [J]." International Journal of Digital Content Technology and Its Application 6.4 (2012): 150-158. 
24. M. Michel, M., Yves, O. Georges, 'Wavelet toolbox TM 4 user's guide. Version 4. Natick, MA: The MathWorks Inc., 2010, pp.4-101-4-115.

25. Vijay, S. G., Kumar, S. H., Srinivasa, P. P., Sriram, S. N., \& Rao, R. B.. "Evaluation of effectiveness of wavelet based denoising schemes using ANN and SVM for bearing condition classification." Computational intelligence and neuroscience 2012 (2012): 16.

26. Breiman, Leo. "Random forests." Machine learning 45.1 (2001): 5-32.

27. Vinay, Vakharia, Gupta Vijay Kumar, and Kankar Pavan Kumar. "Application of chi square feature ranking technique and random forest classifier for fault classification of bearing faults." Proceedings of the 22nd International Congress on Sound and Vibration, Florence, Italy. 2015.

28. Qin, X., Li, Q., Dong, X., \& Lv, S "The Fault Diagnosis of Rolling Bearing Based on Ensemble Empirical Mode Decomposition and Random Forest." Shock and Vibration 2017 (2017).

29. Rajeswari, C., Sathiyabhama, B., Devendiran, S., \& Manivannan, $K$ "Bearing fault diagnosis using wavelet packet transform, hybrid PSO and support vector machine." Procedia Engineering 97 (2014): 1772-1783. 
